\title{
SASHI Suppresses the Proliferation and Invasion of Human Skin Squamous Cell Carcinoma Cells via Inhibiting Akt Cascade
}

This article was published in the following Dove Press journal: OncoTargets and Therapy

\author{
Shang-zhou Chen* \\ Yang Zhang* \\ Shu-ying Lei \\ Fa-qiong Zhou
}

Department of Dermatovenereology, The Central Hospital of Enshi Tujia and Miao Autonomous Prefecture, Enshi 445000, People's Republic of China

*These authors contributed equally to this work
Correspondence: Fa-qiong Zhou Department of Dermatovenereology, The Central Hospital of Enshi Tujia and Miao Autonomous Prefecture, No. 158 Wuyang Road, Enshi 445000, People's Republic of China

Tel +8618672068166

Email1039643683@qq.com
Objective: The SAM- and SH3-domain containing 1 gene (SASH1) has been considered as a tumor suppressor in some cancers. Nevertheless, the effect of SASH1 on the proliferation and invasion of human skin squamous cell carcinoma ( $\mathrm{CSCC}$ ) remains poorly understood. Therefore, the purpose of the present study was to observe the potential role of SASH1 in cSCC and investigate its underlying mechanisms.

Methods: The overexpression of SASH1 was constructed by transfecting the pcDNA3.1/ SASH1 vector into SCL-1 and A431 cells, and SASH1 knockdown was generated by transfecting the SASH1 siRNA into cSCC cells. Then, cell proliferation, invasion, apoptosis, and Akt pathway were observed.

Results: The expression levels of SASH1 mRNA and protein were greatly reduced in cSCC cells. The overexpression of SASH1 inhibited the viability and invasion of cSCC cells, while its knockdown induced the viability and invasion of cSCC cells. The overexpression of SASH1 also suppressed the expression levels of p-Akt and its target genes, including cyclin D1, Bcl-2, and metal matrix proteinase 2(MMP-2). By contrast, SASH1 knockdown exerted the opposite role. Furthermore, inhibition of Akt obviously decreased the inducible effect of cSCC knockdown on the proliferation and invasion of cSCC cells.

Conclusion: Overall, these results found that SASH1 inhibits the proliferation and invasion of cSCC cells via suppressing Akt cascade, indicating a tumor inhibitory effect of SASH1 in cSCC cells.

Keywords: human skin squamous cell carcinoma, SASH1, Akt

\section{Introduction}

In last decades, human skin squamous cell carcinoma (cSCC) and other nonmelanoma skin tumors lead to many tumor-related deaths in the whole world. ${ }^{1}$ Epidemiological survey has reported that more than $20 \%$ of population worldwide could potential occur skin tumor in the life time. ${ }^{2}$ Furthermore, the prevalence of cSCC has been increasing at an amazing rate in the last decade. $^{3}$ The present clinical therapy for cSCC mainly depend on the combinations of surgery, radiotherapy, and/or chemotherapy. ${ }^{4}$ Whereas the prognosis for the advanced and metastatic cSCC is not ideal. ${ }^{5}$ Molecule-targeted treatment is a better option for cSCC, which possibly could help to find novel oncogenic marker for diagnosis and therapy. Moreover, a previous study has reported that SASH1 variants associated with a new genodermatosis with skin carcinoma, and it may be a novel biomarker. ${ }^{6}$ 
SASH1 gene, which belongs to a member of the SLY family of signal adapter proteins, has been found to suppress the proliferation of tumors. ${ }^{7}$ Extensive observations suggested that SASH1 may suppress tumor cell proliferation, migration and invasion in large number of cancer cells. ${ }^{8-10}$ In a recent study, authors have demonstrated that autosomal-recessive SASH1 variants are associated with a new genodermatosis with pigmentation defects, palmoplantar keratoderma and skin carcinoma. ${ }^{6}$ However, the effects of SASH1 on the cell proliferation, migration and invasion of cSCC remain poorly understood. The suppressive role of SASH1 in the protein kinase $\mathrm{B}(\mathrm{Akt})$ has been considered as the underlying mechanism for the SASH-1-stimulated anticancer effect. ${ }^{11,12}$

Akt cascade is an intracellular transduction signaling, which mediates signals from cell membrane receptors to the cytoplasm. ${ }^{13}$ Akt can be induced by some growth factors, such as colony-stimulating factor-1, platelet-derived growth factor, and epidermal growth factor, which are associated with the occurrence of many tumors. ${ }^{14}$ Akt could induce the expression of some cellular proto-oncogenes, such as cyclin D1, B-cell lymphoma protein 2 (Bcl-2), and metal matrix proteinase 2 (MMP-2), which alter the proliferation, cycle, apoptosis, and invasion of tumor cells. ${ }^{15-17}$ SASH1 has been regarded as a negative regulator of Akt transduction. ${ }^{11,12}$ In addition, SASH1 also significantly suppressed the phosphorylation of Akt in gastric cancer cell. ${ }^{18}$ Thus, SASH1 may be a promising molecular target for regulating Akt in the development of novel anti-tumor treatments. However, the role of the Akt-dependent cascade in SASH1-stimulated cell proliferation and invasion of $\mathrm{cSCC}$ cells has never been elucidated.

The purpose of the present study was to observe the related mechanisms of SASH1 on cell proliferation and invasion of cSCC cells.

\section{Materials and Methods Cell Culture}

cSCC cell lines (SCL-1 and A431) and human normal keratinocyte cell line $\mathrm{HaCaT}$ were obtained from Barfield Biology (Wuhan, China). All cell lines were cultured at $37^{\circ}$ $\mathrm{C}$ under $5 \% \mathrm{CO}_{2}$ with Dulbecco's Modified Eagle Medium (DMEM, ScienCell Research Laboratories, USA) containing $10 \%$ fetal bovine serum (FBS, Gibco, USA).

\section{Cell Transfection}

The small interfering RNA (siRNA) for SASH1, Akt and negative control (NC) siRNA were obtained from Barfield
Biology (Wuhan, China) and transfected into cells based on the manufacturer's proposals. The pcDNA/SASH1 expression vector was constructed via inserting SASH1 cDNA into the pcDNA3.1 vector (Eurofins Genomics, Germany). An empty vector was used as a control. The vector was transfected into the cells using Lipofectamine 2000 reagent (Invitrogen) based on the manufacturer's instructions.

\section{Quantitative Real-Time PCR (qRT-PCR)}

Total RNA was extracted from SCL-1 and A431 cells using Trizol reagent (Invitrogen) based on the manufacturer's proposals, and cDNA was synthesized using the QuantiTect Reverse Transcription Kit (Qiagen). qRT-PCR was carried out in a final volume of $10 \mu \mathrm{L}$ reaction mixture, which contained $5 \mu \mathrm{L}$ of SsoFast ${ }^{\mathrm{TM}}$ EvaGreen Supermix (Applied Biosystems), $0.5 \mu \mathrm{L}$ of each primers (SASH1, F: 5'-CAGATCCGGGTGAAGCCAG-3', R: 5'GAGTCCACCACTTGGAATCG-3'; Cyclin D1, F: 5'GAGTAGTGCGAAGCATAGGTCT-3', R: 5'-CTAGCAG AGTAGTCGAGCGC-3'; Bcl-2, F: 5'-TTCTTTGAGTTC GGTGGGG

TC-3', R: 5'-TGCATATTTGTTTGGGGCAGG-3'; MMP-2, F: 5'-TGATCTTGACC

AGAATACCATCGA-3'，R: 5'-GGCTTGCGAGGGA AGAAGTT-3';), $1 \mu \mathrm{L}$ of the cDNA template and $3 \mu \mathrm{L}$ of $\mathrm{ddH}_{2} \mathrm{O}$. PCR amplification was performed using the 7500 Real-Time PCR system (Applied Biosystems, Foster City, CA, USA) and the following cycling conditions: $50^{\circ} \mathrm{C}$ for $2 \mathrm{~min}, 95^{\circ} \mathrm{C}$ for $2 \mathrm{~min}$ followed by 40 cycles of $95^{\circ} \mathrm{C}$ for 3 $\mathrm{s}$ and $60^{\circ} \mathrm{C}$ for $30 \mathrm{~s}$. Reactions were performed in triplicate, and results were analyzed using the $2^{-\Delta \Delta \mathrm{CT}}$ method.

\section{Western Blot Analysis}

Cells were lysed in radioimmunoprecipitation assay (RIPA) buffer (Beyotime, Shanghai, China), centrifuged at $12,000 \mathrm{~g}$ for $12 \mathrm{~min}$ and then heated at $100^{\circ} \mathrm{C}$ for 7 min. Proteins were separated by SDS-PAGE and blotted onto nitrocellulose membranes (Millipore). The membrane was then blocked using 5\% non-fat milk for $1 \mathrm{~h}$ and incubated with primary antibodies (1:1000 dilution) overnight at $4{ }^{\circ} \mathrm{C}$. Primary antibodies (Abcam, UK) against the following proteins were used: SASH1, p-Akt, t-Akt and $\beta$ actin (all dilution: 1:1000). The gel was then incubated with peroxidase-conjugated anti-rabbit $\operatorname{IgG}(1: 10,000$; Abcam). Band intensity was assessed using an enhanced chemiluminescence (Pierce, USA) based on manufacturer protocols. 


\section{Cell Proliferation Assay}

SCL-1 and A431 cell lines were cultured in 96-well plates at a density of $5 \times 10^{3}$ cells/well at $37^{\circ} \mathrm{C}$ for $72 \mathrm{~h}$. Cell Counting Kit-8 (CCK-8) reagent (10 $\mu \mathrm{L}$; Roche Diagnostics, Germany) was then added to each well, and the cells were incubated for $2 \mathrm{~h}$. Absorbance was measured at $450 \mathrm{~nm}$ using a Thermo FC Microplate Reader (Molecular Devices, Biobase, Japan).

\section{Colony Formation Assay}

SCL-1 and A431 cell lines were grown in 6-well culture plates (StemCell Technologies, Canada) at a density of $2 \times 10^{5}$ cells/well for 7 days until colonies were visible. The culture medium was removed and cells were rinsed three times with phosphate-buffered saline (PBS). The colony was fixed and stained with $0.1 \%$ crystal violet (Sigma). The number of colonies was counted under a microscope.

\section{Caspase-3 Activity Assay}

Cell apoptosis was assessed by a caspase-3 activity assay kit (Promega, Mannheim, Germany). Briefly, SCL-1 and A431 cells were degraded using $200 \mu \mathrm{L}$ lysis better (Beyotime, Shanghai, China), then supernatants were recovered after centrifugation and the contents of total protein were detected. The equal amounts of proteins were incubated with $10 \mu \mathrm{L}$ AcDEVD-pNA $(2 \mathrm{mM})$ and $80 \mu \mathrm{L}$ reaction buffer for $3 \mathrm{~h}$. The absorbance was measured at $405 \mathrm{~nm}$ using a Thermo FC Microplate Reader (Molecular Devices, Biobase, Japan).

\section{In vitro Cell Invasion Assay}

Cell invasion was assessed by 24-well transwell units (Coming, NY, USA) with a polycarbonate filter (pore size, $8 \mu \mathrm{m}$ ) coated with extracellular matrix gel (Millipore Chemicon, MA, USA) to form a continuous thin layer. SCL-1 and A431 cells $\left(2 \times 10^{5}\right.$ per well) were grown in the upper chamber and $500 \mu \mathrm{L}$ DMEM was added to the lower chamber. The cells were then cultured for $24 \mathrm{~h}$. Cells on the upper surface of the filter were completely removed by wiping with a cotton swab, and then the filters were fixed in 5\% methanol and stained with Wright-Giemsa stain (Invitrogen, Carlsbad, CA, USA). Cells that had invaded the Matrigel and reached the lower surface of the filter were counted using a light microscope (BD Biosciences, Heidelberg, Germany).

\section{Statistical Analysis}

Differences between different groups were evaluated for significance using Student's $t$-test. A $p$-value of $<0.05$ was regarded as statistically significant. Results are shown as the mean \pm standard deviation.

\section{Results}

\section{SASHI Is Downregulated in cSCC Cells}

To explore the underlying expression levels of SASH1 in cSCC, we measured the expression levels of SASH1 mRNA and protein in HSSCC cells using RT-PCR and Western blotting, respectively. Our results (Figure 1) showed that SASH1 is downregulated in, both at the mRNA and the protein levels, in two adherently cultured cSCC lines compared to one atypical keratinocyte line (HaCaT).
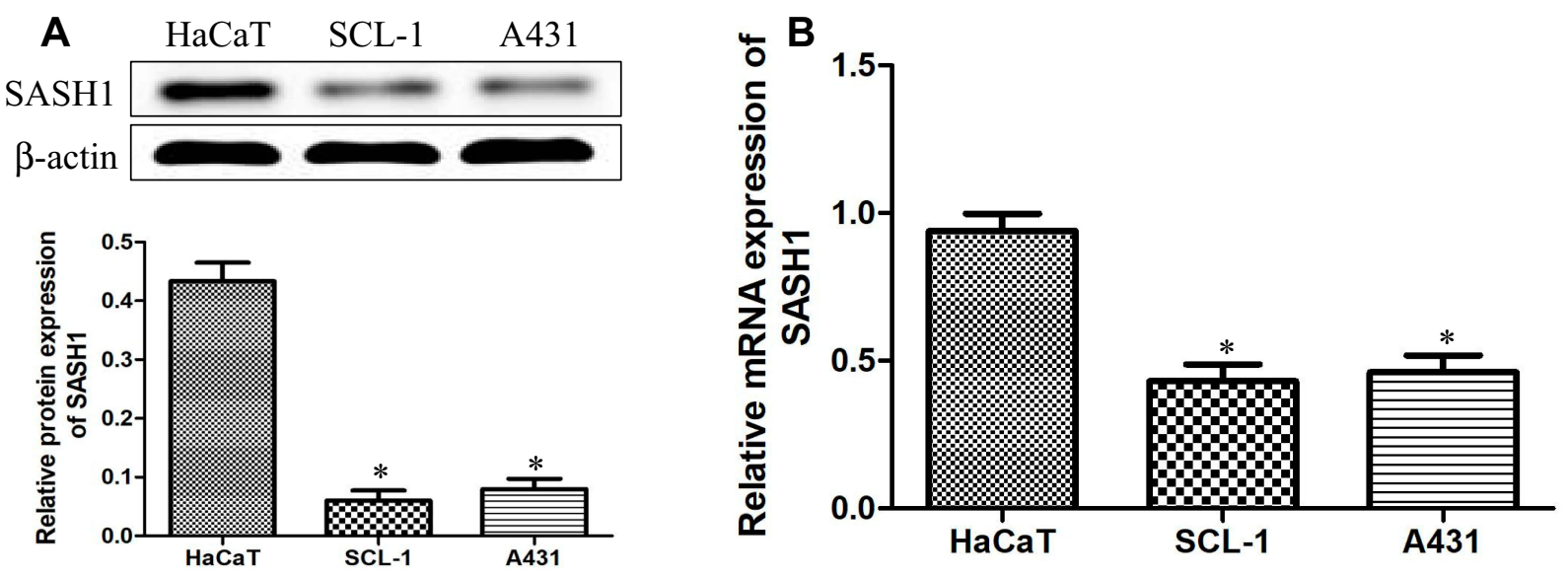

Figure I Downregulation of SASHI in HSSCC cell lines. The protein (A) and mRNA (B) expressions of SASHI in HaCaT, SCL-I, and A43I cells were measured using Western blotting analysis and RT-PCR, respectively. $\mathrm{HaCaT}$ cells regarded as a control, compared with $\mathrm{HaCaT}$ cells. $* P<0.05$. 
A
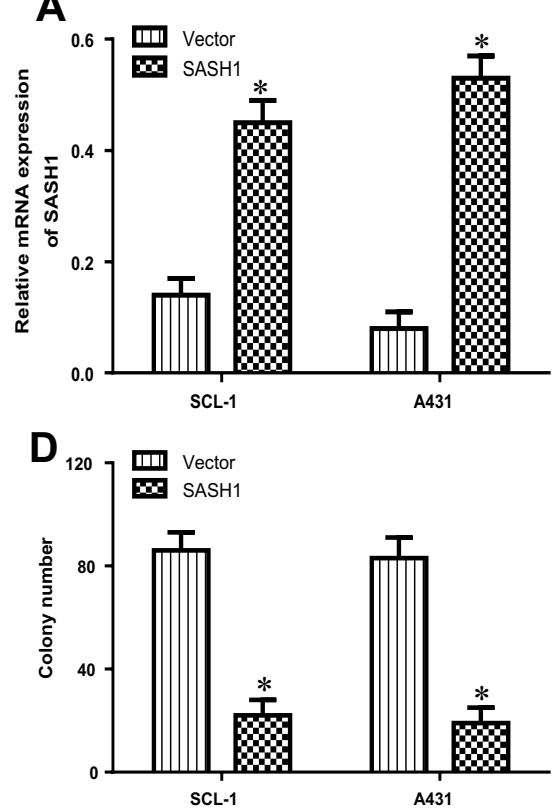

B
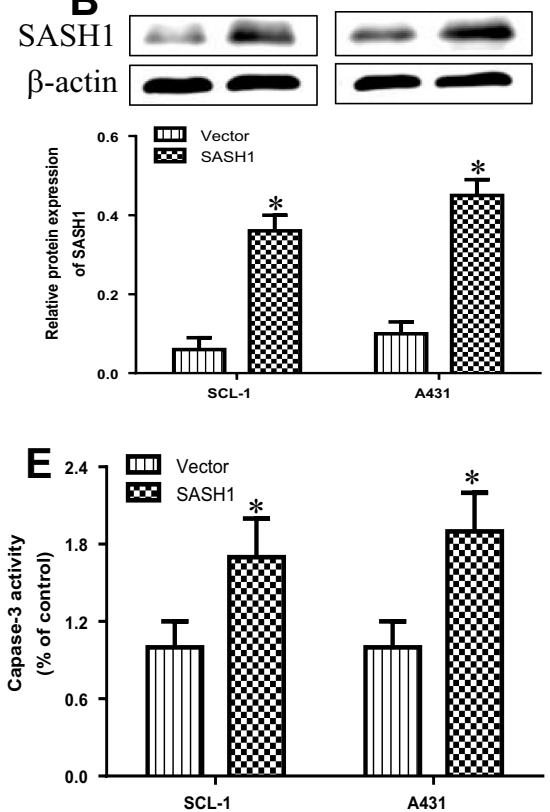

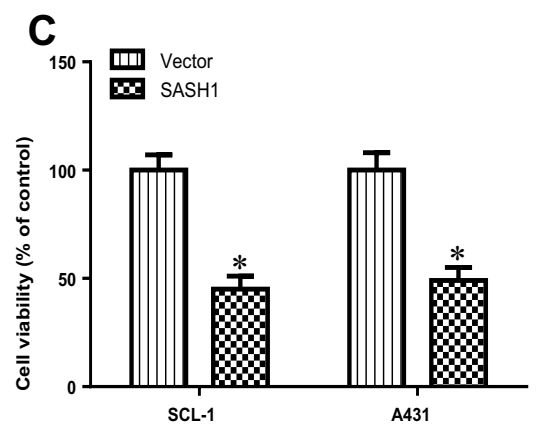

$\mathbf{F}$

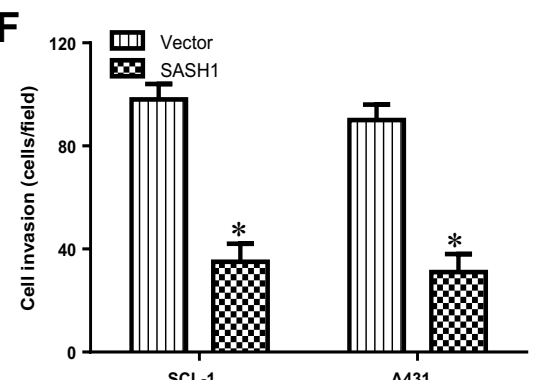

Figure 2 SASHI overexpression suppresses the cell viability and invasion of HSSCC cells. HSSCC cell lines transfecting with pcDNA3. I/SASHI vector or empty vector for $48 \mathrm{~h}$ were constructed. The expression levels of SASHI mRNA $(\mathbf{A})$ and protein (B) were determined using RT-PCR and Western blotting, respectively. (C) Cell viability was measured using the CCK-8 assay. (D) Colony formation was evaluated using the colony formation assay. (E) Cell apoptosis was measured using the caspase-3 activity test. (F) Cell invasion was assessed by the transwell invasion assay. Compared with vector group. $* P<0.05$.
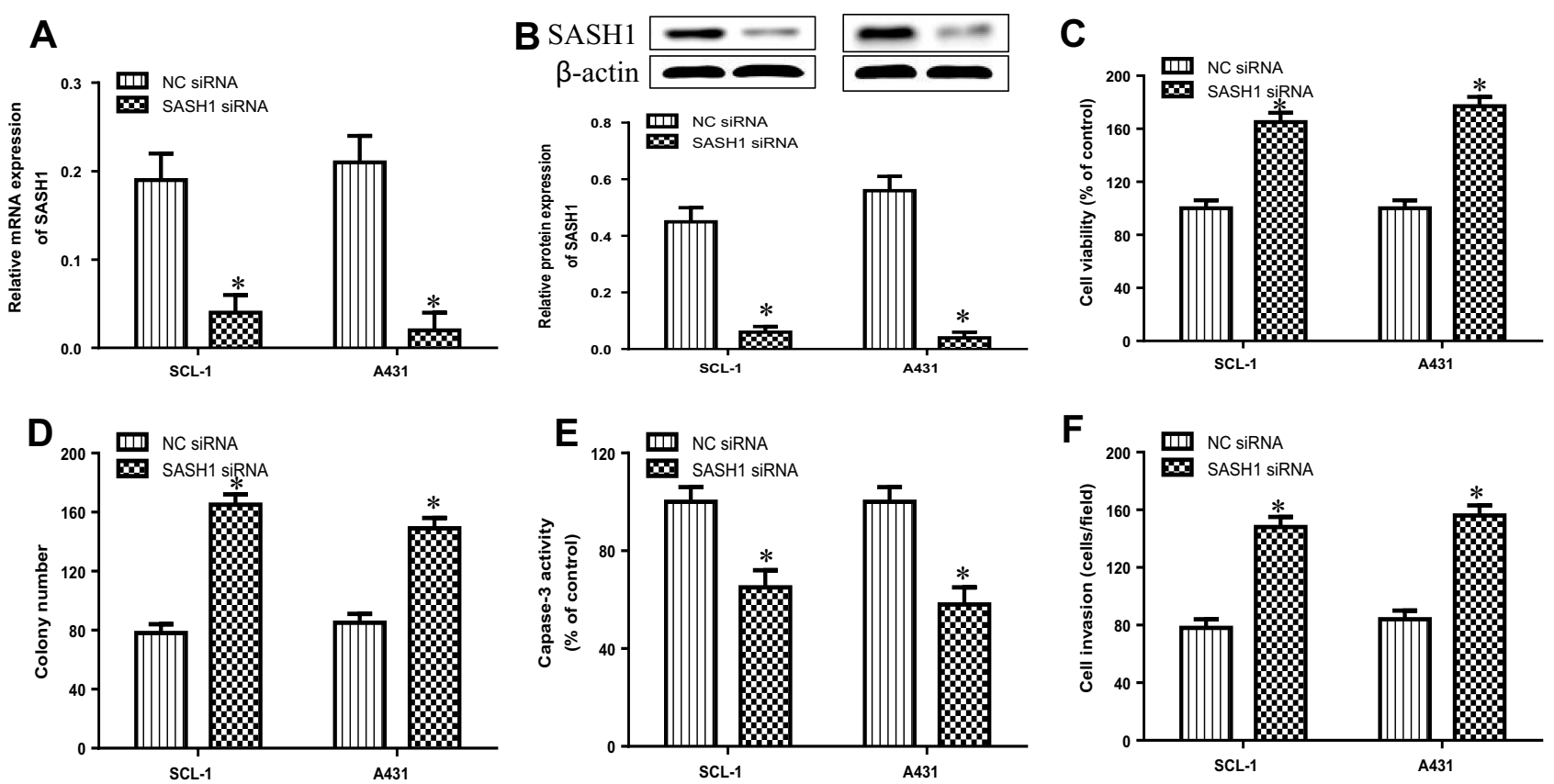

Figure 3 SASHI knockdown induces the cell viability and invasion of HSSCC cells. HSSCC cell lines transfecting with SASHI siRNA or NC siRNA for 48 h were constructed. The expression levels of SASHI mRNA (A) and protein (B) were determined using RT-PCR and Western blotting, respectively. (C) Cell viability was measured using the CCK-8 assay. (D) Colony formation was evaluated using the colony formation assay. (E) Cell apoptosis was measured using the caspase-3 activity test. (F) Cell invasion was assessed by the transwell invasion assay. Compared with NC siRNA group. $* P<0.05$. 


\section{Upregulation of SASHI Suppresses the Proliferation and Invasion of cSCC Cell Lines}

To observe the biological function of SASH1 in cSCC, we analyzed the impacts of SASH1 overexpression on the proliferation and invasion of cSCC cell lines by transfecting the pcDNA3.1/SASH1 vector into SCL-1, and A431 cells. The mRNA (Figure 2A) and protein (Figure 2B) expressions were increased after transfecting with the pcDNA3.1/ SASH1 vector when compared to the empty vector. The overexpression of SASH1 inhibited the cell viability of SCL-1 and A431 cells (Figure 2C). Furthermore, SASH1 upregulation also suppressed the colony formation of SCL-1 and A431 cells (Figure 2D). In addition, we further determined the impact of SASH1 upregulation on the apoptosis of SCL-1 and A431 cells using caspase-3 activity analysis. SASH1 overexpression also stimulated the activity of caspase- 3 in HSSCC cells (Figure 2E). In addition, the impacts of SASH1 overexpression on cell invasion were further explored using transwell invasion assay. As displayed in Figure 2F, SASH1 overexpression inhibited the invasion of SCL-1 and A431 cells.

\section{Knockdown of SASHI Induces the Proliferation and Invasion of cSCC Cell Lines}

To further explore the biological function of SASH1 in cSCCC, we observed the effects of SASH1 downregulation
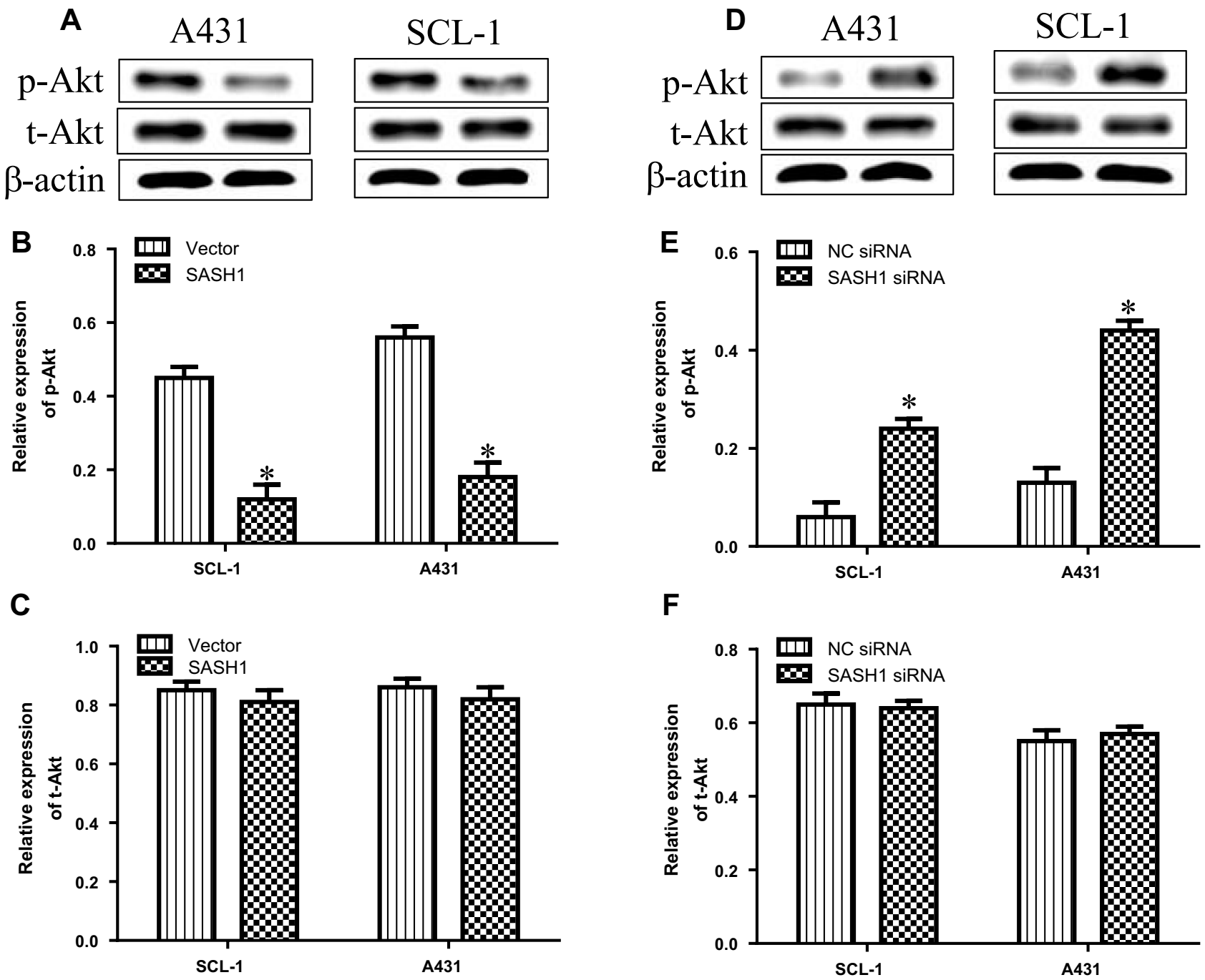

Figure 4 SASHI mediates the alterations in the phosphorylation levels of Akt in HSSCC cells. (A) Western blotting analyses of p-Akt and t-Akt expressions in SCL-I and A43I cells transfecting with pcDNA3.I/SASHI vector or empty vector for $48 \mathrm{~h}$. Quantitative analyses of p-Akt (B) and t-Akt (C) in HSSCC cells. Compared with vector group, $* P<0.05$. (D) Western blotting analyses of $\mathrm{p}-\mathrm{Akt}$ and t-Akt expressions in SCL-I and A43I cells transfecting with SASHI siRNA or NC siRNA for $48 \mathrm{~h}$. Quantitative analyses of $\mathrm{p}$-Akt $(\mathbf{E})$ and t-Akt $(\mathbf{F})$ in HSSCC cells. Compared with NC siRNA group. $* P<0.05$. 
on the proliferation and invasion of cSCC cell lines by transfecting the SASH1 siRNA into SCL-1 and A431 cells. The mRNA (Figure 3A) and protein (Figure 3B) expressions were reduced after transfecting with the SASH1 siRNA when compared to the negative control (NC, empty vector) siRNA. The knockdown of SASH1 increased the cell viability of SCL-1 and A431 cells (Figure 3C). Furthermore, SASH1 knockdown also promoted the colony formation of SCL-1 and A431 cells (Figure 3D). As shown in Figure 3E, SASH1 knockdown decreased the activity of caspase-3 in cSCC cells. In addition, as displayed in Figure 3F, SASH1 knockdown inhibited the invasion of SCL-1 and A431 cells. Taken together, our data indicate that the downregulation of SASH1 may contribute to the occurrence of cSCC.

\section{SASHI Alters the Phosphorylation of Akt Protein in CSCC Cells}

Previous observation have reported that SASH1 may suppress hepatocarcinoma cell invasion and metastasis via down-regulating the Akt cascade in vivo and in vitro. ${ }^{11}$ To observe whether SASH1 alters Akt activity, we explored the impact of SASH1 on the phosphorylation of activated Akt in cSCC cells. Our results suggested that the overexpression of SASH1 inhibited the levels of p-Akt in SCL-1 and A431 cells, with no significant impact on the $\mathrm{t}$-Akt expression (Figure 4A-C). On the other hand, knockdown of SASH1 significantly induced the levels of p-Akt in SCL-1 and A431 cells (Figure 4D-F). Collectively, our data indicate that SASH1 functions as a negative regulator of Akt in cSCC cells.

\section{SASHI Regulates the Expressions of Akt Target Genes in cSCC Cells}

We further determined the impacts of SASH1 on the transcription of Akt target genes, such as cyclin D1, Bcl-2, and MMP-2. Our data suggested that SASH1 upregulation inhibited the mRNA expression levels of cyclin D1, Bcl-2, and MMP-2 in SCL-1 and A431 cells (Figure 5A-C). In addition, downregulation of SASH1 induced the mRNA expression levels of cyclin D1, Bcl-2, and MMP-2 in SCL-1 and A431 cells (Figure 5D-F). Taken together, these findings suggest that SASH1 reversely regulates Akt pathway.

\section{Inhibition of Akt Improves the Impact of SASHI Knockdown in cSCC Cells}

To further verify that SASH1 has an antitumor activity via Akt cascade, we depleted the expression of Akt in SASH1 siRNA transfected cells. These results showed that the depletion of Akt by siRNA (Figure 6A and B) decreased the inducible effect of SASH1 knockdown on the proliferation (Figure 6C) and invasion (Figure 6D) of SCL-1 and A431 cells. These findings indicate that SASH1
A

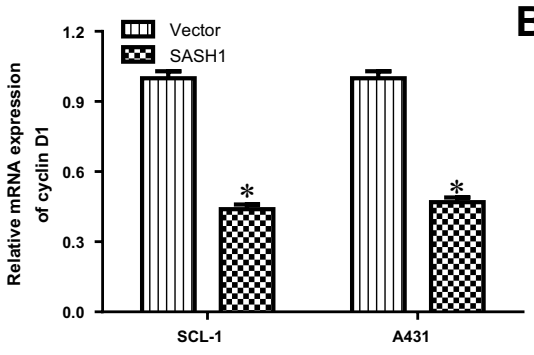

D

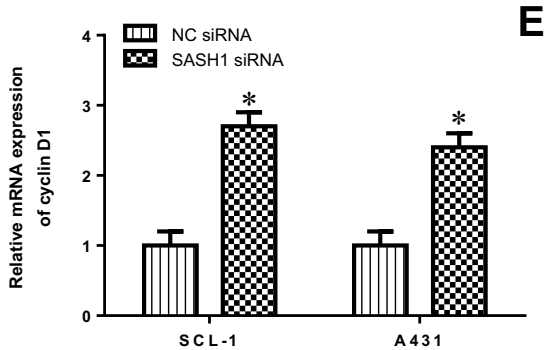

B

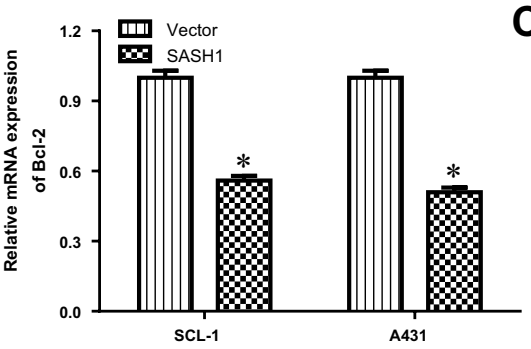

E

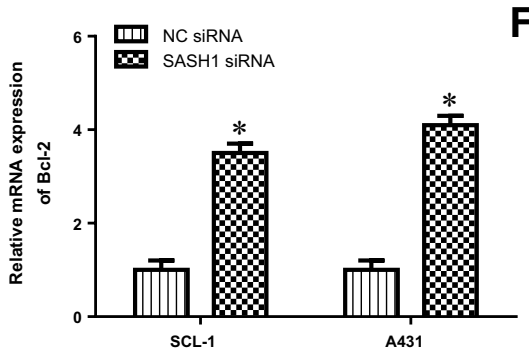

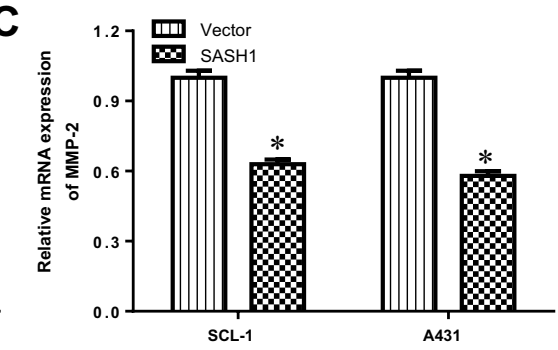

$\mathbf{F}$

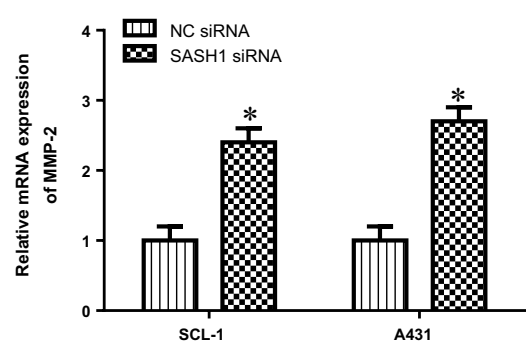

Figure 5 SASHI regulates the mRNA expression levels of SASHI target genes in HSSCC cells. The mRNA expressions of cyclin DI (A), Bcl-2 (B), and MMP-2 (C) in SCL-I and A43I cells transfecting with pcDNA3. I/SASHI vector or empty vector for $48 \mathrm{~h}$ were determined using RT-PCR. Compared with vector group, *P<0.05. The mRNA expressions of cyclin DI (D), Bcl-2 (E), and MMP-2 (F) in SCL-I and A43I cells transfecting with SASHI siRNA or NC siRNA for $48 \mathrm{~h}$ were measured by RT-PCR. Compared with NC siRNA group. $* P<0.05$. 

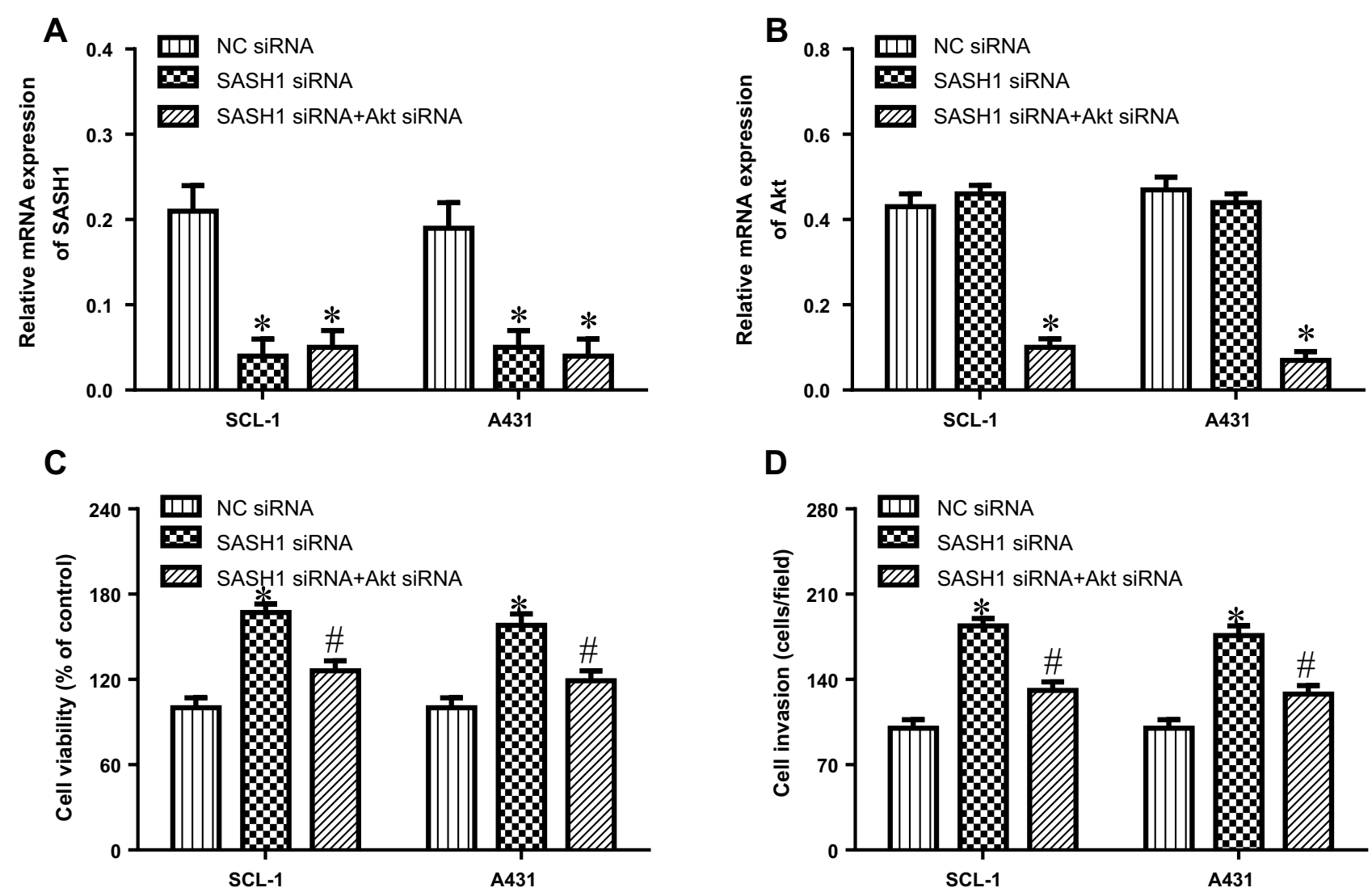

Figure 6 Inhibition of Akt improves the impacts of SASHI knockdown on the proliferation and invasion of HSSCC cells. SCL-I and A43I cell lines were transfected with SASHI siRNA and Akt siRNA for $48 \mathrm{~h}$. The mRNA expressions of SASHI (A) and Akt (B) were measured using RT-PCR. (C) The proliferation of SCL-I and A43I cell lines were determined using CCK-8 assay. (D) Cell invasion was observed using the transwell invasion assay. Compared with NC siRNA group. $* P<0.05$; compared with SASHI siRNA group, ${ }^{\#} P<0.05$.

suppresses the proliferation and invasion of cSCC cells, partly through Akt pathway.

\section{Discussion}

In recent years, the role of SASH1 in tumor occurrence has been well-verified. Nevertheless, the effect of SASH1 on cSCC is still poorly understood. We have found that the expression level of SASH1 in cSCC cells is downregulated, indicating that it serves as a cancer suppressor. We also found that the up-regulation of SASH1 suppresses the cell viability and invasion of cSCC cell lines via decreasing the activation of Akt pathway. These results show that SASH1 is closely associated with the occurrence of cSCC.

SASH1 has been reported to repress the growth of multiple tumors. ${ }^{8-10}$ Low expression or knockdown of SASH1 promote epithelial-mesenchymal transition (EMT), further resulting in an aggressive, invasive phenotype with elevated chemoresistance. ${ }^{7}$ SASH1 deficiency was also associated with the increased proliferation and aggressiveness of oesophageal squamous cell carcinoma (ESCC) cells. ${ }^{19}$ In addition, the inhibition of SASH1 by miR-17 is involved in the increased cell proliferation, migration and the decreased cell apoptosis in osteosarcoma cells. ${ }^{20}$ Thus, SASM1 is closely correlated with the development and progression of tumors and could be regarded as a promising marker for diagnosis and prognosis of malignant neoplasm. The mutation of SASH1 is also related to the cellular process of apoptosis. ${ }^{21}$ Whereas the effect of SASH1 on cSCC is still unclear. A recent research demonstrated that SASH1 mutations are associated with the occurrence and development of skin carcinoma. ${ }^{6}$ In our research, we showed that SASH1 was dramatically decreased in cSCC cell lines. Up-regulation of SASH1 suppresses the proliferation and invasion of SCL-1 and A431 cells, while SASH1 down-regulation exert the opposite roles. This research conforms a cancer inhibitory effect of SASH1 in cSCC.

SASH1 has been identified as an inhibitor of Akt to repress the phosphorylation of Akt in tumor cells. ${ }^{11,12}$ 
Similar to these results, we also found that SASH1 is a negative regulator of Akt in cSCC cells. Our results suggested that overexpression of SASH1 dramatically suppress the phosphorylation of Akt. Moreover, SASH1 also suppressed the expression of Akt downstream target genes, including cyclin D1, Bcl-2, and MMP-2, associated with the development of cSCC. The induced cell proliferation and invasion under SASH1 knockdown is abolished by inhibition of Akt, indicating that SASH1 performs roles in cSCC possibly via regulating the activation of Akt. Taken together, these findings suggest that the SASH1/Akt cascade participants in the development of cSCC, providing a new insight into the molecular pathogenesis of cSCC.

Akt serves as an important regulator of the development and progression of $\mathrm{cSCC}^{22}$ Overexpression of $\mathrm{p}$-Akt is found in the tissues of skin cancer, and high expression of $\mathrm{p}$-Akt is associated with the invasion and metastasis of cSCC. $^{23}$ Thus, Akt could be used as a therapeutic molecular target for cSCC. In our research, we have conformed SASH1 is a negative regulator of Akt in cSCC cells. The downregulation of SASH1 leads to the elevated activation of Akt in cSCC. Therefore, suppressing Akt by SASH1 might represent a potential therapeutic mean for cSCC. This conclusion was supported by the findings that the inhibitor of PI3K/Akt/ mTOR pathway (LY3023414) could suppress the growth of cSCC cells in vitro and in vivo. ${ }^{24}$ Our data suggest that SASH1 suppresses the proliferation and invasion of cSCC cells correlated with the downregulation of Akt-mediated the expression of cyclin D1, Bcl-2, and MMP-2. Akt has been demonstrated to induce cell cycle progression via regulating the levels of cyclin D1, cyclin B1, cyclin E, and c-Myc. ${ }^{15}$

\section{Conclusions}

Collectively, we found that SASH1 has a tumor inhibitory effect in cSCC. Our research shows that SASH1 is downregulated in cSCC cells and that overexpression of SASH1 inhibits the proliferation and invasion of cSCC cells correlated with downregulation of Akt pathway. This research provides a new insight into the molecular pathogenesis of cSCC and shows that regulating the expression of SASH1 might result in a novel therapeutic strategy for cSCC.

\section{Acknowledgments}

The authors thank the Central Laboratory of The Central Hospital of Enshi Tujia and Miao Autonomous Prefecture. Shang-zhou Chen and Yang Zhang are co-first authors for this study.

\section{Disclosure}

All authors declare that there is no conflict of interest.

\section{References}

1. Martin A, Salvador F, Moreno-Bueno G, et al. Lysyl oxidase-like 2 represses Notch1 expression in the skin to promote squamous cell carcinoma progression. EMBO J. 2015;34(8):1090-1109. doi:10.15 252/embj.201489975

2. Teepen JC, Kok JL, Kremer LC, et al. Long-term risk of skin cancer among childhood cancer survivors: a DCOG-LATER cohort study. J Natl Cancer Inst. 2019;5:219-231.

3. Faltaous AA, Leigh EC, Ray P, et al. A rare transformation of epidermoid cyst into squamous cell carcinoma: a case report with literature review. Am J Case Rep. 2019;20(6):1141-1143. doi:10.12659/AJCR.912828

4. Soydemir GP, Kandaz M, Melikoğlu M. The results of radiotherapy for squamous cell carcinomas of the skin. Dermatol Ther. 2019;8(6): e13058.

5. Bashline B. Skin cancer: squamous and basal cell carcinomas. FP Essent. 2019;481(7):17-22.

6. Courcet JB, Elalaoui SC, Duplomb L, et al. Autosomal-recessive SASH1 variants associated with a new genodermatosis with pigmentation defects, palmoplantar keratoderma and skin carcinoma. Eur J Hum Genet. 2015;23(7):957-962. doi:10.1038/ejhg.2014.213

7. Franke FC, Müller J, Abal M. The tumor suppressor SASH1 interacts with the signal adaptor CRKL to inhibit epithelial-mesenchymal transition and metastasis in colorectal cancer. Cell Mol Gastroenterol Hepatol. 2018;7(6):33-53. doi:10.1016/j.jcmgh.2018.08.007

8. Li Z, Ni J, Song D, Ding M. Regulatory mechanism of microRNA-128 in osteosarcoma tumorigenesis and evolution through targeting SASH1. Oncol Lett. 2018;15(10):8687-8694. doi:10.3892/ ol.2018.8397

9. Zhou N, Liu C, Wang X, Mao Q, Jin Q, Li P. Downregulated SASH1 expression indicates poor clinical prognosis in gastric cancer. Hum Pathol. 2018;74(6):83-91. doi:10.1016/j.humpath.2018.01.008

10. Xie J, Zhang W, Zhang J, Lv Q-Y, Luan Y-F. Downregulation of SASH1 correlates with poor prognosis in cervical cancer. Eur Rev Med Pharmacol Sci. 2017;21(3):3781-3786.

11. Sun CY, Zhang ZH, He P, Zhou Y, Xie X. Involvement of PI3K/Akt pathway in the inhibition of hepatocarcinoma cell invasion and metastasis induced by SASH1 through downregulating Shh-Gli1 signaling. Int J Biochem Cell Biol. 2017;89(6):95-100. doi:10.1016/ j.biocel.2017.06.006

12. Sun D, Zhou R, Liu H, Sun W, Dong A, Zhang H. SASH1 inhibits proliferation and invasion of thyroid cancer cells through PI3K/Akt signaling pathway. Int J Clin Exp Pathol. 2015;8(4):12276-12283.

13. Pitaloka DMI, Ko CH, Lin MT, Yeh S-L, Yeh C-L. Glutamine administration promotes hepatic glucose homeostasis through regulating the PI3K/Akt pathway in high-fat diet-induced obese mice with limb ischemia. Nutr Res. 2019;68(4):45-53. doi:10.1016/j.nutres.2019.05.008

14. Chen J, Hao Y, Chen J, et al. Colony stimulating factor-1 receptor promotes proliferation, migration and invasion in the human nasopharyngeal carcinoma $6-10 \mathrm{~B}$ cell line via the phosphoinositide 3-kinase/Akt pathway. Oncol Lett. 2018;16(3):1205-1211. doi:10. 3892/ol.2018.8750

15. Zong D, Jiang N, Xu JH. ZNF488 is an independent prognostic indicator in nasopharyngeal carcinoma and promotes cell adhesion and proliferation via collagen IV/FAK/AKT/Cyclin D1 pathway. Cancer Manag Res. 2019;11(3):5871-5882. doi:10.2147/CMAR. $\mathrm{S} 200001$

16. Huang SH, Tseng JC, Lin CY. Rooibos suppresses proliferation of castration-resistant prostate cancer cells via inhibition of Akt signaling. Phytomedicine. 2019;64(5):153-168. doi:10.1016/j.phymed.2019.153 068 
17. Wang $\mathrm{Z}, \mathrm{Xu} \mathrm{H}$, Zhang J. Basic fibroblast growth factor blockade enhances lung cancer cell invasion by activating the AKT/MMP-2/ VEGF pathway. Basic Clin Pharmacol Toxicol. 2019;21(3):309-321.

18. Zong W, Yu C, Wang P. Overexpression of SASH1 inhibits TGFB1-induced EMT in gastric cancer cells. Oncol Res. 2016;24 (9):17-23. doi:10.3727/096504016X14570992647203

19. Zhu Y, Ma Y, Peng H. MiR-130b promotes the progression of oesophageal squamous cell carcinoma by targeting SASH1. $J$ Cell Mol Med. 2019;23(3):93-103. doi:10.1111/jcmm.13887

20. Wu D, Zhang H, Ji F. MicroRNA-17 promotes osteosarcoma cells proliferation and migration and inhibits apoptosis by regulating SASH1 expression. Pathol Res Pract. 2019;215(3):115-120. doi:10.1016/j.prp.2018.10.012

21. Tashjian RZ, Granger EK, Farnham JM. Genome-wide association study for rotator cuff tears identifies two significant single-nucleotide polymorphisms. J Shoulder Elbow Surg. 2016;25(3):174-179. doi:10.1016/j.jse.2015.07.005
22. Ci C, Wu C, Lyu D. Down-regulation of kynureninase restrains cutaneous squamous cell carcinoma proliferation and represses PI3K/AKT pathway. Clin Exp Dermatol. 2019;16(3):34-46.

23. Chamcheu JC, Roy T, Uddin MB. Role and therapeutic targeting of the PI3K/Akt/mTOR signaling pathway in skin cancer: a review of current status and future trends on natural and synthetic agents therapy. Cells. 2019;8(1):E803. doi:10.3390/cells8080803

24. Zou Y, Ge M, Wang X. Targeting PI3K-AKT-mTOR by LY3023414 inhibits human skin squamous cell carcinoma cell growth in vitro and in vivo. Biochem Biophys Res Commun. 2017;490(2):385-392. doi:10.1016/j.bbrc.2017.06.052

\section{Publish your work in this journal}

OncoTargets and Therapy is an international, peer-reviewed, open access journal focusing on the pathological basis of all cancers, potential targets for therapy and treatment protocols employed to improve the management of cancer patients. The journal also focuses on the impact of management programs and new therapeutic

Submit your manuscript here: https://www.dovepress.com/oncotargets-and-therapy-journal agents and protocols on patient perspectives such as quality of life, adherence and satisfaction. The manuscript management system is completely online and includes a very quick and fair peer-review system, which is all easy to use. Visit http://www.dovepress.com/ testimonials.php to read real quotes from published authors. 